\title{
Elliptical Multipole Wiggler Beamlines at the Advanced Photon Source
}

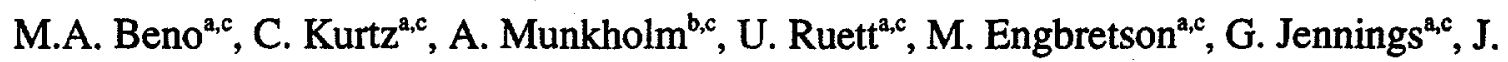
Linton $^{\text {a,c }}$, G. S. Knapp ${ }^{a, c}$, and P.A. Montano ${ }^{a, c, d}$

${ }^{2}$ Materials Science Division, ${ }^{b}$ Chemistry Division and ${ }^{c}$ Basic Energy Sciences Synchrotron Radiation Center, Argonne National Laboratory, Argonne, IL 60439 USA

d Department of Physics, University of Illinois at Chicago, Chicago, IL USA

Materials Science Division

Argonne National Laboratory

Argonne, IL 60439

The submitted manuscript has been created by
the University of Chicago as Operator of Argonne
National Laboratory ("Argonne") under Contract
No. W-31-109-ENG-38 with the U.S. Department
of Energy. The U.S. Government retains for
itself, and others acting on its behalf, a paid-up,
non exclusive, irrevocable worldwide license in
said article to reproduce, prepare derivative
works, distribute copies to the public, and
perform publicly and display publicly, by or on
behalf of the Government.

September 2000

/jc

\section{Distribution:}

1-2. PRS, 203

3. J. M. Gibson

4. P. A. Montano

5. F. Y. Fradin

6. Office of Science

7. J. Coble

8. Authors

Submitted for publication in Nuclear Instrument and Methods, Section A, from the $7^{\text {th }}$ International Synchrotron Radiation Instrumentation conference (SRI 2000) held from August 20-25, 2000 in Berlin, Germany.

This work is supported by the US Department of Energy, Office of Basic Energy Sciences under contract No. W-31-109-ENG-38. 


\section{DISCLAIMER}

This report was prepared as an account of work sponsored by an agency of the United States Government. Neither the United States Government nor any agency thereof, nor any of their employees, make any warranty, express or implied, or assumes any legal liability or responsibility for the accuracy, completeness, or usefulness of any information, apparatus, product, or process disclosed, or represents that its use would not infringe privately owned rights. Reference herein to any specific commercial product, process, or service by trade name, trademark, manufacturer, or otherwise does not necessarily constitute or imply its endorsement, recommendation, or favoring by the United States Government or any agency thereof. The views and opinions of authors expressed herein do not necessarily state or reflect those of the United States Government or any agency thereof. 


\section{DISCLAIMER}

Portions of this document may be illegible in electronic image products. Images are produced from the best available original document. 


\title{
ELLIPTICAL MULTIPOLE WIGGLER BEAMLINES AT THE ADVANCED PHOTON SOURCE
}

\author{
M. A. Beno ${ }^{a, c}$, C. Kurtz ${ }^{a, c}$, A. Munkholm ${ }^{b, c}, U$. Rutt t, , M. Engbretson ${ }^{a, c}$, G. Jennings ${ }^{a, c}$, \\ J. Linton ${ }^{a, c}$, G. S. Knapp ${ }^{a, c}$ and P. A. Montano ${ }^{a, c, d}$
}

${ }^{a}$ Materials Science Division, ${ }^{b}$ Chemistry Division and ${ }^{c}$ Basic Energy Sciences

Synchrotron Radiation Center, Argonne National Laboratory, Argonne, IL, 60439, USA

${ }^{d}$ Department of Physics, University of Illinois at Chicago, Chicago, IL, USA

Keywords: Synchrotron Radiation, Monochromator, Compton Scattering, High Energy Diffraction

PACS.07.85.Qe, PACS.41. 50.th

Corresponding Author: Mark A. Beno, Bldg 433, Argonne National Laboratory

9700 S. Cass Ave, Argonne, IL, 60439, USA

Phone:(630)252-3507, Fax:(630)252-0365, Email: beno@anl.gov

\begin{abstract}
The Basic Energy Sciences Synchrotron Radiation Center Collaborative Access Team has built three independent beamlines, which simultaneously utilize the $\mathrm{x}$-ray radiation from an elliptical multipole wiggler, located at Sector 11 of the Advanced Photon Source. This insertion device produces circularly polarized $x$-rays on-axis and linearly polarized $x$-rays above and below the ring plane. The lower linearly polarized radiation is used in the monochromatic 11ID-D station for scattering and spectroscopy experiments in the 5 to $40 \mathrm{keV}$ range. The on-axis circularly polarized photons are used for magnetic Compton scattering experiments in the 11ID-B station. The upper linearly polarized radiation is utilized by the high-energy diffraction station, 11ID-C. We report here on the beamline optics and experimental station equipment.
\end{abstract}

\section{Introduction}

Part of the mission of the Basic Energy Sciences Synchrotron Radiation Center Collaborative Access Team (BESSRC-CAT) was to construct and instrument a 
beamline for magnetic Compton scattering experiments. To this end the Advanced Photon Source (APS) in collaboration with BESSRC-CAT designed and built an elliptical multipole wiggler (EMPW) [1]. This device, which has a maximum critical energy of $32 \mathrm{keV}$ and a total power output of $\sim 8 \mathrm{~kW}$, uses conventional permanent magnets to provide the vertical field $\left(K \mathrm{~K}_{\max }=14\right)$. Electromagnets produce the horizontal field $\left(\mathrm{Kx}_{\max }=1.4\right)$, which is switchable so that the helicity of the on-axis circular polarization can be flipped at a rate up to $10 \mathrm{~Hz}$. The calculated radiation profile from the EMPW under normal operating conditions $(\mathrm{Ky}=14, \mathrm{Kx}=1.0)$ is shown in Figure 1. The 11-ID EMPW provides circular polarization on axis while producing intense lobes of linearly polarized $x$-rays above and below the ring plane. This radiation profile provides the opportunity for a beamline design which uses not only the on-axis circularly polarized radiation for which the insertion device was designed, but also takes advantage of the high photon fluxes available in the off-axis linearly polarized lobes. The EMPW beamline design, that we have constructed, uses the single insertion device to provide photons independently to three different experimental stations. Each station has a specific experimental mission and therefore different challenges in optics design.

\section{Beamline Design}

Figure 2 is a schematic view of the beamline layout. The first component in the white light enclosure 11ID-A (first optics enclosure, FOE) is a water cooled copper mask, which limits the horizontal beam size incident on the beamline optics to $1 \mathrm{mrad}$ horizontal and a total power of $\sim 4 \mathrm{~kW}$. The FOE contains three monochromators, one for each of the branch beamlines, and bremsstrahlung shielding. The D-branch monochromatic shutter and focusing mirrors are also positioned in the FOE. A horizontal fan of three shielded beam transport pipes exits the downstream end of the 11ID FOE, which delivers beam to three end-stations.

The straight through beam, which originates from the lower linearly polarized radiation lobe, is used for scattering and spectroscopy in the 11ID-D experimental 
station. The D-branch beamline has a fixed-exit, double crystal monochromator with a $20 \mathrm{~mm}$ vertical offset. Its monochromatic beam passes above the $\mathrm{B}$ and $\mathrm{C}$ branch monochromators. Pt or Pd coated toroidal focusing mirrors positioned downstream of the monochromator provide a high photon flux $\left(5 \times 10^{12}\right.$ photons $/ \mathrm{s}$ at $\left.10 \mathrm{keV}\right)$ in a $1 \times$ $0.5 \mathrm{~mm}^{2}$ mirror focus. The $800 \mathrm{~mm}$ long Pt-coated mirror has a sagittal radius of 73.3 $\mathrm{mm}$ and is intended to operate at an incident angle of $2.4 \mathrm{mrad}$ providing a high energy cutoff of $\sim 36 \mathrm{keV}$. The $770 \mathrm{~mm}$ long Pd mirror has a sagittal radius of 122 $\mathrm{mm}$ and is designed to operate at an incidence of $4 \mathrm{mrad}$ yielding a high-energy cutoff at $\sim 16 \mathrm{keV}$.

The two horizontally deflecting branches provide monochromatic $\mathrm{x}$-rays to the 11ID-C and 11ID-B experimental stations. The C-branch beamline uses the upper linear lobe of $x$-rays, which is used for for high energy $x$-ray experiments [2]. The Bbranch beamline uses the on-axis circularly polarized beam and is designed for magnetic Compton scattering experiments . Both the 11ID-C and 11ID-B monochromators are located in a helium filled chamber and are identical in design. The beam is monochromatized by a horizontally deflecting Laue silicon crystal at a fixed Bragg angle $\theta$, which is $1.9^{\circ}$ and $3.8^{\circ}$ for the $\mathrm{C}$ and $\mathrm{B}$-branch, respectively.

An enclosure, that houses a high-power high-repetition rate laser, is located immediately down stream of the 11ID-D station. The laser beam can be directed into the 11ID-D station for spectroscopy or scattering experiments involving optically excited states of molecules or materials.

\section{Monochromators}

The D-branch monochromator is a liquid nitrogen cooled, fixed-exit, double crystal $\operatorname{Si}(220)$ monochromator, which is capable of operations from to $4^{\circ}-55^{\circ}$ allowing experiments from approximately 5 to $40 \mathrm{keV}$. The design is similar to the BESSRC bending magnet (12BM) monochromator detailed in these proceedings[3]. Major changes from the 12BM monochromator design are: (i) liquid nitrogen cooling 
for the first crystal by use of a first crystal design and cooling scheme identical to that used in the BESSRC undulator monochromator (12ID) [4], (ii) translation of the center of rotation of the first crystal from the middle of the top surface to the downstream tip of the crystal, (iii) reduction of the crystal offset to $20 \mathrm{~mm}$, and (iv) elimination of the horizontal slide in the Cowan-Golovchenko linkage by use of a long second crystal. Because of the high critical energy of the EMPW (32 keV), large amounts of Compton scattering are generated in the monochromator chamber, which puts a high heat load on the second crystal. To maintain the temperature of the second crystal in a range where fixed exit operation is possible, the last two changes were required in order to allow cooling of the $150 \mathrm{~mm}$ long second crystal by gold foil strips linked to the first crystal liquid nitrogen cooling. Translation of the center of rotation was necessary to allow scanning of the monochromator energy without obstructing either the on-axis circular polarization or the upper linear lobe of radiation. A detailed schematic drawing of the D-branch monochromator is shown in Fig. 3.

The $\mathrm{C}$ branch beamline uses a horizontally deflecting Laue monochromator with a fixed Bragg angle of $\theta=1.9^{\circ}$. At this angle, the interchangeable $1 \mathrm{~cm}^{3}$ cubes of annealed silicon [5] ( $\mathrm{Si}(111), \mathrm{Si}(220)$, or $\mathrm{Si}(311))$ provide fixed energies of 60 $\mathrm{keV}, 98 \mathrm{keV}$ or $114 \mathrm{keV}$. The photon flux is $2 \times 10^{12}$ photons $/ \mathrm{s}$ in a $2.5 \times 3.5 \mathrm{~mm}^{2}$ spot at $98 \mathrm{keV}$. The B branch beamline uses $\mathrm{Si}(220), \mathrm{Si}(400)$, or $\mathrm{Si}(711)$ crystals at a fixed Bragg angle of $3.8^{\circ}$, which provide fixed energies of 49,69 , or $123 \mathrm{keV}$ and their higher harmonics. The annealed $\mathrm{Si}$ crystals $(1 \mathrm{~cm}$ on an edge) provide a bandwidth for the monochromator of $\mathrm{dE} / \mathrm{E} \approx 5 \times 10^{-3}$, which is well matched to the energy resolution of the solid state detectors used for magnetic Compton scattering experiments in the 11ID-B station. A photon flux of $1 \times 10^{12}$ photons $/ \mathrm{sec}$ is observed at $100 \mathrm{keV}$ with $\mathrm{P}_{\mathrm{c}}=50 \%$. Both $\mathrm{B}$ and $\mathrm{C}$ branch monochromators are mounted on a Huber 410 rotation table ( $\theta$ motion) and a motorized goniometer head ( $\chi$ motion). Translation of the goniometer head is used to switch between up to 3 monochromator 
crystals. Cooling of the monochromator crystals is provided by suspending the $\mathrm{Si}$ crystals in a In-Ga eutectic mixture, which fills a turn water-cooled tray. The total power incident on the $\mathrm{B}$ and $\mathrm{C}$-branch monochromator crystals is limited by the use of a $1.5 \mathrm{~mm}$ thick Copper filter.

\section{Experimental Stations}

The 11ID-D experimental station is equipped with a kinematic optical table (1.2 $\mathrm{m} \mathrm{x}$ $1.8 \mathrm{~m}$ ) for spectroscopy experiments and a Huber 8-circle Psi diffractometer for scattering experiments. The optical table is capable of accurate motions with loads in excess of $200 \mathrm{~kg}$ and can be translated to give from $0-400 \mathrm{~mm}$ of beam clearance, which allows for large cryostats or heavy magnets to be accommodated. The diffractometer is mounted on a high load capacity $(>2000 \mathrm{~kg})$ table, which provides accurate cross-beam and instrument height adjustments. A large vertical travel range is important for both tables because of the large height differential $(90 \mathrm{~mm}$ ) between the beam reflected from the Pt and Pd mirrors. Ion chambers, single and multielement solid state $(\mathrm{Ge})$ detectors and photomultiplyer detectors are available. Ancillary equipment available includes a DISPLEX system, and a He-flow Cryostat. Data acquisition can be accomplished using SPEC [6], or by using EPICS through a Windows based software [7] or an IGOR interface [8].

The 11ID-C experimental station is instrumented with a vertical triple crystal diffractometer [2,9], that also can be configured for low resolution experiments in the horizontal scattering plane. The diffractometer is designed for heavy cryostats or magnets $(>200 \mathrm{~kg}$ ) and can be equipped with a DISPLEX or helium flow cryostat. The station has a special single element Ge solid state detector designed for high energy ( $>100 \mathrm{keV})$ operation. Beamline control is accomplished with the IGOREPICS interface [7].

The 11ID-B experimental station is instrumented for Magnetic Compton scattering experiments [10] with an Oxford superconducting magnet mounted on a kinematic table. The magnet and cryostat can provide fields up to 8 tesla and 
temperatures as low as $1.2 \mathrm{~K}$. A 1 tesla electromagnet, which is capable of switching polarities at a rate of up to $10 \mathrm{~Hz}$, is also available. Compton scattering experiments use a three element solid state detector $(\mathrm{Ge})$ with $1 \mathrm{~cm}$ thick elements for high energy operation. As in the $\mathrm{C}$ station, data acquisition is done using EPICS through an IGOR interface [7].

\section{ACKNOWLEDGMENTS}

Work at Argonne National Laboratory is supported by the US Department of Energy (DOE), Office of Basic Energy Sciences; Division of Material Sciences, under contract W-31-109-ENG-38.

\section{REFERENCES}

1. P. A. Montano, G. S. Knapp, G. Jennings, E. Gluskin, E. Trakhtenberg, I. B. Vasserman, P. M. Ivanov, D. Frachon, E. R. Moog, L. R. Turner, G. K. Shenoy, M. J. Bedzyk, M. Ramanathan, M. A. Beno, and P. L. Cowan, Rev. Sci. Instr., 66(2) (1995) 1839-1841.

2. U. Rütt, J. R. Schneider, M. A. Beno, G. S. Knapp and P. A. Montano, Proc. SPIE, 3448 (1998) 132.

3. M. A. Beno, M. Engbretson, G. Jennings, G. S. Knapp, J. Linton, C. Kurtz, U. Rütt and P. A. Montano, "BESSRC-CAT Bending Magnet Beamline at the Advanced Photon Source", these proceedings.

4. G. S. Knapp, M. A. Beno, C. S. Rogers, C. L. Wiley and P. L. Cowan, Rev. Sci. Inst., 65 (1994) 2792.

5. S. Keitel, U. Rütt, M. A. Beno, and J. R. Schneider , "Optimization of monochromator crystals by in situ studies of the annealing process of $\mathrm{Cz}$-grown silicon" in 1998 HASYLAB Annual Report.

6. Certified Scientific Software, Cambridge, MA.

7. M. Engbretson, Rapid Application Development (RAD) Software for Synchrotron Beamline Control, in preparation.

8. G. Jennings, Synchrotron Beamline and Instrument Control with IGOR, in preparation.

9. U. Rütt, M. A. Beno, J. Strempfer, G. Jennings and P. A. Montano

"Diffractometer for High Energy X-Rays at the APS", these proceedings.

10. P. A. Montano, Y. Li, U. Rütt, M. A. Beno, G. Jennings and C. W. Kimball, Proc. SPIE 3773 (1999) 262. 
Figure Captions:

Figure 1.

Contour plot of the calculated EMPW spectral flux (photons $/ \mathrm{mm}^{2} \cdot \mathrm{s}$ ) at the white-light mask (27 $\mathrm{m}$ from the source). The central shaded area corresponds to the aperture of the mask ( $8 \mathrm{~mm}$ vertical by $30 \mathrm{~mm}$ horizontal).

Figure 2.

Top view of the $11 \mathrm{ID}$ beamlines. The first optics enclosure, $11 \mathrm{ID}-\mathrm{A}$, contains all the white-light components. Photons for the D-branch beamline are passed through a vertical double crystal monochromator and subsequent focussed by a toroidal mirror in the A-hutch. The 11ID-B and $\mathrm{C}$ stations use horizontal diffracting Laue monochromators at fixed angles of $3.8^{\circ}$ and $1.9^{\circ}$, respectively.

\section{Figure 3.}

Schematic of the liquid nitrogen cooled D-branch Si(220) monochromator. Fixed exit operation is maintained by the roller driven vertical motion of the second crystal kinematic mount and by the use of a long second crystal. When the energy is changed, the beam diffracted from the first crystal walks along the surface of the second crystal. 


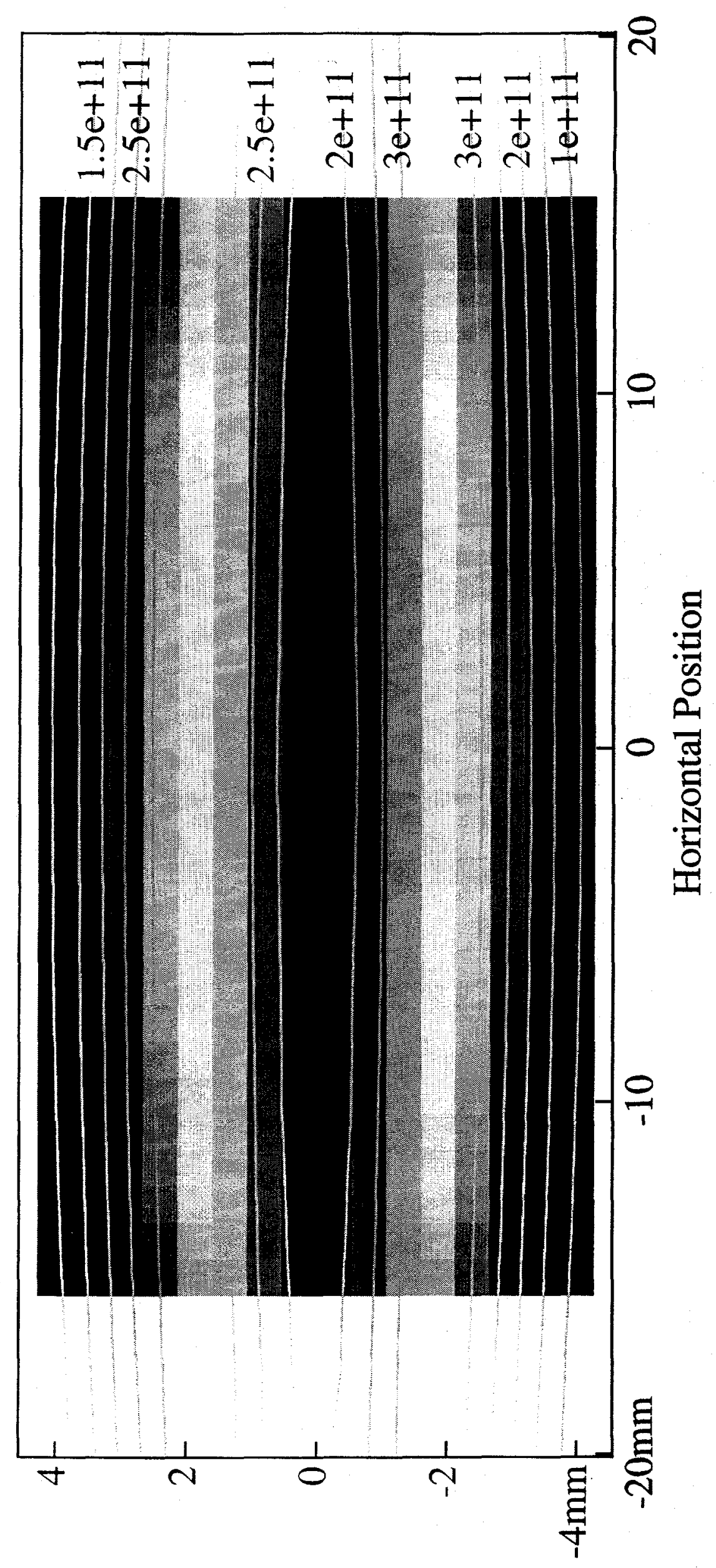

薏 


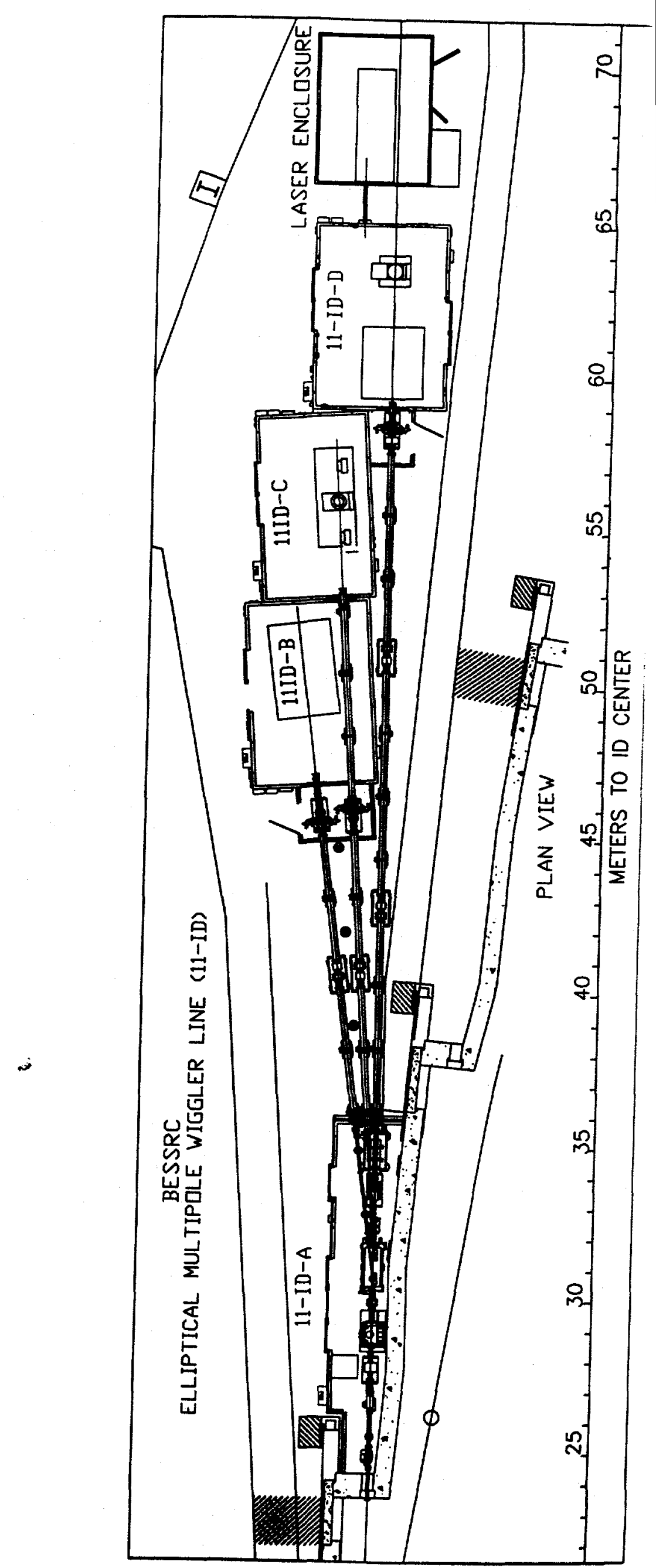




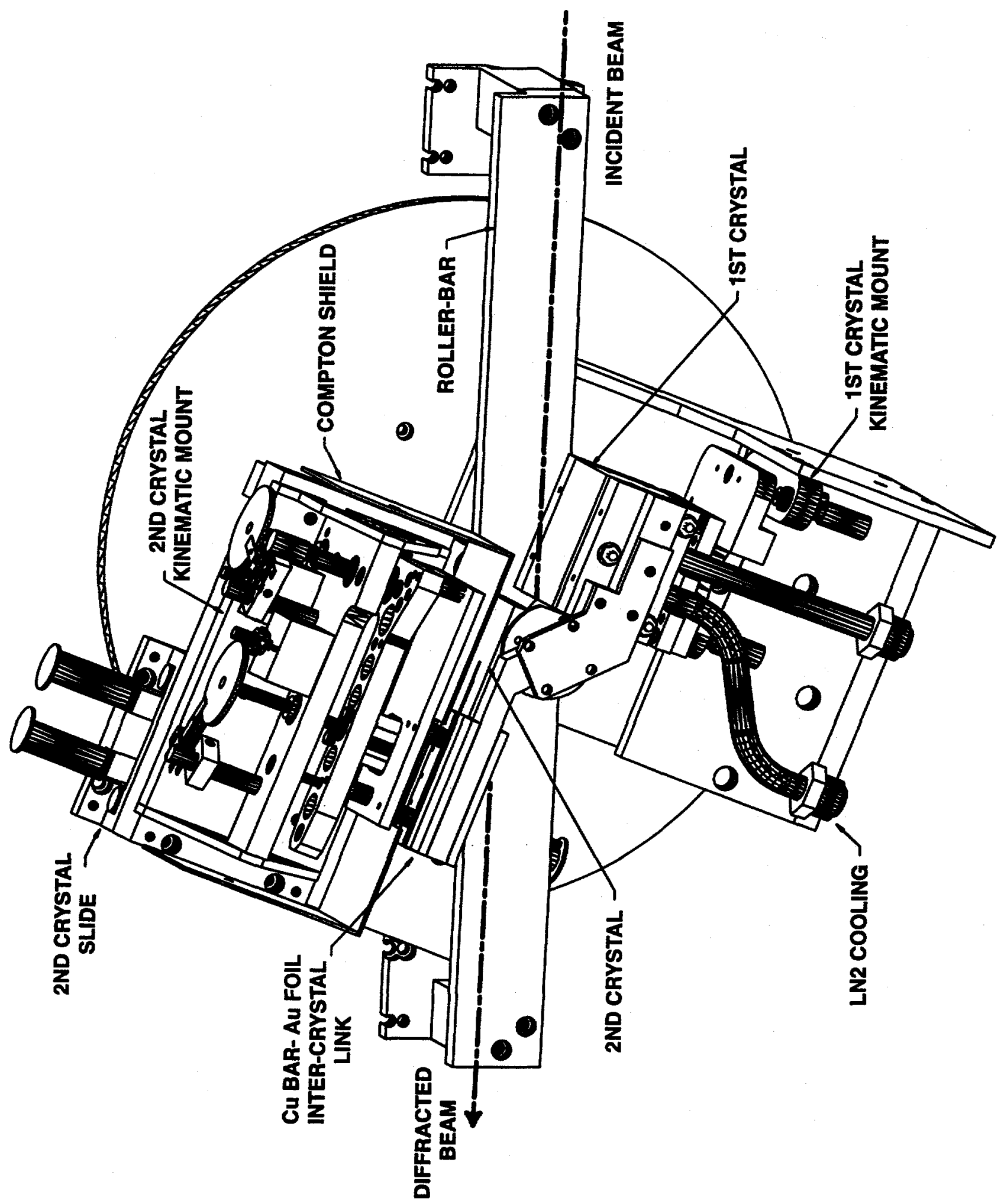

